\title{
Robotic radical hysterectomy: a new standard of care?
}

\section{Pedro T Ramirez}

Department of Gynecologic Oncology, The University of Texas MD Anderson Cancer Center, Houston, TX, USA = Tel.: +1 7137455498 m Fax: +1 7137927586 = peramire@mdanderson.org

Evaluation of: Ko EM, Muto MG, Berkowitz RS, Feltmate CM: Robotic versus open radical hysterectomy: a comparative study at a single institution. Gynecol. Oncol. 111 (3), 425-430 (2008). Minimally invasive surgery has been shown to be associated with a number of advantages to the patient, such as lower blood loss, lower transfusion rates, lesser requirements of pain medications, quicker return of bowel function and a shorter recovery period, as well as earlier resumption of daily activities. More recently, robotic surgery has become increasingly popular among gynecologic oncologists who perform minimally invasive surgery. Robotic surgery has allowed surgeons to be able to offer patients the same benefits of laparoscopy, while being able to perform procedures that require advanced surgical skills. This retrospective study showed that robotic radical hysterectomy was associated with less blood loss and a shorter length of stay compared with the open approach. In addition, the authors showed that intraoperative and postoperative complications were comparable.

A recent publication by Ko and colleagues [1] compared robotic versus open radical hysterectomy in women diagnosed with early cervical cancer. A number of studies have demonstrated the benefits of minimally invasive surgery in gynecologic oncology. In patients with early-stage cervical cancer undergoing radical hysterectomy, laparoscopic surgery has been shown to be safe and feasible [2-8]. However, laparoscopic surgery is associated with a number of disadvantages. Among these, the most common include prolonged operative times, a steep learning curve, limited two-dimensional visualization, instruments with a restricted range of motion, and higher costs than equivalent procedures performed by laparotomy. More recently, robotic surgery has become increasingly popular among gynecologic oncologists.

Sert and Abeler were the first to report on a patient who had undergone a robotic radical hysterectomy [9]. Since their original report, a number of other small series, totaling slightly over 100 patients, have been published [10-13]. Each of these series has shown that robotic surgery has an advantage over laparoscopy and laparotomy, with robotic surgery patients having less blood loss, a lower transfusion rate and shorter length of hospitalization.

The article by Ko and colleagues [1], published in the Gynecologic Oncology journal, is yet another in a series of important contributions to the minimally invasive literature in our field. The objective of the study was to compare the shortterm surgical outcomes of patients undergoing robotic radical hysterectomy and compare those with patients who underwent a similar procedure by laparotomy. The authors found that the mean operative time was significantly longer for robotic radical hysterectomy than for open radical hysterectomy ( 4.5 vs $3.4 \mathrm{~h}, \mathrm{p}=0.0002$ ). The mean estimated blood loss was significantly less (82 vs $667 \mathrm{ml}, \mathrm{p}<0.0001$ ) for the robotic group than for the open radical hysterectomy group. As anticipated, the mean length of stay was significantly shorter for the robotic radical hysterectomy group (1.7 vs 4.9 days, $\mathrm{p}<0.0001$ ).

There are several important observations in this study that ought to be highlighted. First, the study reports on the experience of a group of surgeons who transitioned from laparotomy to a robotic approach in performing a procedure that demands a high level of skill and training. The majority of the current series comparing robotic radical hysterectomy to either laparoscopy or laparotomy report on the experience of surgeons that have already had extensive laparoscopic experience and, therefore, one might argue that the favorable numbers seen in those reports are reflective of the fact that these surgeons have already surpassed their minimally invasive learning curve when performing this particular procedure. Although surgeons in the group reporting this study had previous laparoscopic experience, the data reported in this article is a direct comparison of robotic surgery to laparotomy without a transition period through laparoscopy. Second, there were no differences in patient demographics with regard to age, stage or body mass index between the robotic surgery group and the laparotomy group. Third, the authors confirmed that with increasing experience, the
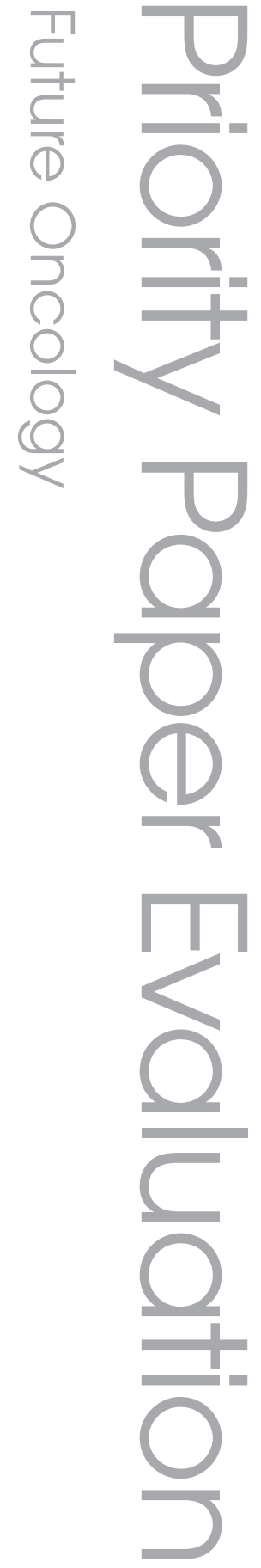

\section{Keywords}

cervical cancer - laparoscopy = minimally invasive surgery $=$ robotic radical hysterectomy

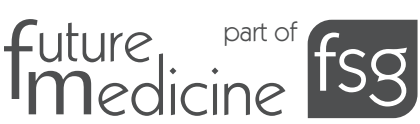


intraoperative time decreased, further supporting an improvement in their skills and operative techniques as their experience progressed. Fourth, although this was their initial experience with robotic surgery, their rate of intraoperative complications did not differ from the laparotomy group. Fifth, the authors reported no conversions to laparotomy. Sixth, although there was no information reported in this study regarding oncologic parameters such as parametrial length or vaginal cuff length, the authors did note that there were no differences between the two groups with regard to the number of lymph nodes retrieved or rate of positive margins.

In analyzing this study, several criticisms ought to be highlighted. This is a retrospective, nonrandomized study of selected patients who were compared with historical controls. The number of patients reported is a small series of patients, with only 16 patients in the robotic radical hysterectomy group. In addition, there is no information provided on the length of follow-up for the patients included in this study. Similar to other series reported, the data is the result of procedures performed by only two surgeons. This study also shows that the overall complication rate in the robotic radical hysterectomy group was the same as that seen in the laparotomy group. One would expect that the robotic approach would decrease the complication rate. Nevertheless, in other comparative series, the robotic approach has consistently decreased the rate of wound and bowel complications as compared with laparotomy. One of the greatest criticisms of robotic surgery has been the cost of this system. In this study, there is no cost-effective analysis, and thus the reader may be left wondering: what is the impact on the healthcare cost of performing robotic surgery versus laparotomy in patients requiring radical hysterectomy?

The authors of this study ought to be congratulated for their contribution by sharing their initial experience in robotic surgery. Based on these results, there is a clear advantage to robotic surgery as it reduces blood loss, transfusion rate and length of hospitalization. Based on the preliminary data that has been published, albeit flawed by small number of patients and short follow-up, there does not appear to be a compromise in oncologic outcomes in the robotic surgery groups, as the number of lymph nodes appears equivalent or higher with the robotic approach. In addition, some reports have shown that the parametrial sizes or vaginal margins are not compromised in patients undergoing robotic radical hysterectomy.

One very important issue that fails to be mentioned in any of the robotic radical hysterectomy series thus far reported is the impact of implementing robotic surgery in fellowship training programs. The studies reported to date are all reflective of the experience of either a single surgeon or a limited number of faculties reporting on their personal experience. We must all make a concerted effort to continue pursuing proficiency in robotic surgery so that we may surpass our initial learning curve and therefore start integrating our fellows-in-training in the critical portions of the procedures.

Many questions still remain regarding the impact on overall survival, long-term complications and, in particular, quality-of-life assessment. A prospective, randomized trial has been initiated at The University of Texas MD Anderson Cancer Center (TX, USA), comparing open radical hysterectomy versus laparoscopic or robotic radical hysterectomy in patients with early-stage cervical cancer. This is a multi-institutional international trial expected to accrue a total of 740 patients. Whether robotic radical hysterectomy should be considered the standard approach in the management of patients with early-stage cervical cancer remains a topic of debate. Investigators should continue to report on their experience so that we may ultimately be able to address this question with definitive and supportive data.

\section{Executive summary}

- Robotic surgery is associated with lower blood loss and shorter length of hospitalization.

Advantages of robotic surgery include: better visualization, instruments with higher dexterity, precise surgeon motioning and improved surgeon ergonomics.

- The main disadvantage of robotic surgery is the cost of the equipment.

- Conversion rates to open surgery from robotic surgery are the same or less than with laparoscopic surgery.

- Intraoperative complications with robotic surgery are the same or less than with open or laparoscopic surgery.

- Robotic radical hysterectomy appears to be equivalent to open or laparoscopic radical hysterectomy in overall oncologic outcomes.

A prospective randomized international trial is currently being conducted evaluating open versus minimally invasive surgery in patients undergoing a radical hysterectomy. 


\section{Financial \& competing interests \\ disclosure}

The author has no relevant affiliations or financial involvement with any organization or entity with a financial interest in or financial conflict with the subject matter or materials discussed in the manuscript. This includes employment, consultancies, honoraria, stock ownership or options, expert testimony, grants or patents received or pending, or royalties.

No writing assistance was utilized in the production of this manuscript.

\section{Bibliography}

1. Ko EM, Muto MG, Berkowitz RS, Feltmate CM: Robotic versus open radical hysterectomy: A comparative study at a single institution. Gynecol. Oncol. 111(3), 425-430 (2008).

2. Canis M, Mage G, Wattiez A et al.: Does endoscopic surgery have a role in radical surgery of cancer of the cervix uteri? J. Gynecol. Obstet. Biol. Reprod. (Paris) 19, 921 (1990).

3. Nezhat CR, Burrell MO, Nezhat FR et al.: Laparoscopic radical hysterectomy with para-aortic and pelvic node dissection. Am. J. Obstet. Gynecol. 166, 864-865 (1992).

4. Spirtos NM, Eisenkop SM, Schlaerth JB et al: Laparoscopic radical hysterectomy (Type III) with aortic and pelvic lymphadenectomy in patients with stage I cervical cancer: surgical morbidity and intermediate follow-up. Am. J. Obstet. Gynecol. 187, 340-348 (2002).

5. Abu-Rustum NR, Gemignani ML, Moore K et al.: Total laparoscopic radical hysterectomy with pelvic lymphadenectomy using the argon-beam coagulator: pilot data and comparison to laparotomy. Gynecol. Oncol. 91, 402-409 (2003).

6. Gil-Moreno A, Diaz-Feijoo B, Roca I et al: Total laparoscopic radical hysterectomy with intraoperative sentinel node identification in patients with early invasive cervical cancer. Gynecol. Oncol. 96, 187-193 (2005).

7. Ramirez PT, Slomovitz BM, Soliman PT et al.: Total laparoscopic radical hysterectomy and lymphadenectomy: the M.D. Anderson Cancer Center experience. Gynecol. Oncol. 102, 252-255 (2006).

8. Frumovitz M, dos Reis R, Sun CC et al:: Comparison of total laparoscopic and abdominal radical for patients with early-stage cervical cancer. Obstet.Gynecol. 110(1), 96-102 (2007).

9. Sert BM, Abeler VM: Robotic-assisted laparoscopic radical hysterectomy (Piver Type III) with pelvic node dissection case report. Eur. J. Gynaecol. Oncol. 27(5), 531-533 (2006).

10. Sert B, Abeler V: Robotic radical hysterectomy in early-stage cervical carcinoma patients, comparing results with total laparoscopic radical hysterectomy cases. The future is now? Int. J. Med. Robot. 3(3), 224-228 (2007).

11. Kim YT, Kim SW, Hyung WJ et al:: Robotic radical hysterectomy with pelvic lymphadenectomy for cervical carcinoma: a pilot study. Gynecol. Oncol. 108(2), 312-316 (2008).

12. Magrina JF, Kho RM, Weaver AL et al:: Robotic radical hysterectomy: comparison with laparoscopy and laparotomy. Gynecol. Oncol. 109(1), 86-91 (2008).

13. Boggess JF, Gehrig PA, Cantrell L et al:: A case-control study of robot-assisted Type III radical hysterectomy with pelvic lymph node dissection compared with open radical hysterectomy. Am. J. Obstet. Gynecol. 199, 357-359 (2008).

\section{Affiliation}

- Pedro T Ramirez, MD

Associate Professor, Director of Minimally Invasive Surgical Research \& Education, Department of Gynecologic Oncology, The University of Texas MD Anderson Cancer Center, Houston, TX, USA 